\title{
Anethole Supplementation During Oocyte Maturation Improves In Vitro Production of Bovine Embryos
}

\author{
Naiza A. R. Sá, MSc', Luís A. Vieira, PhD², \\ Anna Clara A. Ferreira, MSc', Jesús Cadenas, PhD', \\ Jamily B. Bruno, PhD', Carolina Maside, PhD², \\ Francisca G. C. Sousa, PhD', Francielli W. S. Cibin, PhD $^{3}$, \\ Benner G. Alves, PhD ${ }^{4}$, Ana Paula R. Rodrigues, PhD', \\ José H. Leal-Cardoso, PhD ${ }^{5}$, Eduardo L. Gastal, PhD $^{6}$, \\ and José R. Figueiredo, PhD'
}

\begin{abstract}
Oxidative stress is one of the most detrimental factors that affect oocyte developmental competence and embryo development in vitro. The impact of anethole supplementation to in vitro maturation (IVM) media on oocyte maturation and further bovine in vitro embryo production was investigated. Oocytes of slaughterhouse-derived bovine ovaries were placed in IVM with anethole at different concentrations of 30 (AN30), 300 (AN300), and $2000 \mu \mathrm{g} / \mathrm{mL}$ (AN2000), or without (control treatment). The oocytes were assessed for maturation rates, and for reactive oxygen species (ROS) and ferric reducing antioxidant power (FRAP) levels, and mitochondrial membrane potential. Embryo development was assessed by cleavage and blastocyst rates, and embryo cell number. The percentage of metaphase II oocytes were similar among the treatments (range, $77 \%-96 \%$ ). Anethole at $300 \mu g / m L$ was the only treatment that yielded higher cleavage and embryo development (morula and blastocyst) rates compared to the control treatment. The ROS production in the oocytes after maturation did not differ among treatments. However, oocytes treated with anethole at $300 \mu \mathrm{g} / \mathrm{mL}$ had higher $(P<.05)$ FRAP and mitochondrial membrane potential compared to the control treatment. Furthermore, AN300 treatment increased $(P<.05)$ the average number of total cells in blastocysts compared to the control and AN30 treatments. The use of anethole at $300 \mu \mathrm{g} / \mathrm{mL}$ during IVM is suggested to improve the quantity and quality of bovine embryos produced in vitro. The beneficial effects of anethole on embryonic developmental competence in vitro seems to be related to its capacity to regulate the redox balance and improve mitochondrial function in oocytes and embryos.
\end{abstract}

\section{Keywords}

antioxidant, anethole, ROS, mitochondria, oocyte, embryo

\section{Introduction}

Although in vitro embryo production (IVP) protocols have progressed substantially in recent years, the IVP rates are still lower than their in vivo counterparts. ${ }^{1}$ In a comprehensive recent review article on cattle, ${ }^{2}$ the following general outcomes were reported for IVP: $70 \%$ to $90 \%$ maturation rate; $50 \%$ to $80 \%$ fertilization rate, including 1 to 2-cell stage; and blastocyst production rate of $20 \%$ to $40 \%$. However, although promising alternatives to improve IVP in cattle have been recently reported, using an antioxidant substance (eg, lycopene), the blastocyst production rate was still low (eg, $\leq 29 \%){ }^{3}$ For that reason, different approaches have been used to improve the efficiency of bovine IVP technology, enhancing, consequently, the production of metaphase II (MII) oocytes, and good-quality embryos. Improvement of culture media for both oocyte in

\footnotetext{
' Laboratory of Manipulation of Oocytes and Preantral Follicles (LAMOFOPA), Faculty of Veterinary Medicine, State University of Ceara, Fortaleza, Ceara, Brazil

${ }^{2}$ Department of Physiology, Veterinary Faculty, University of Murcia, Murcia, Spain

${ }^{3}$ Laboratory of Reproduction Biotechnology (Biotech), Campus Uruguaiana, Federal University of Pampa, Uruguaiana, Rio Grande do Sul, Brazil

${ }^{4}$ Laboratory of Reproductive Biology, Institute of Biomedical Sciences, Federal University of Uberlandia, Uberlandia, Minas Gerais, Brazil

${ }^{5}$ Laboratory of Electrophysiology (LEF), Superior Institute of Biomedical Sciences, State University of Ceara, Fortaleza, Ceara, Brazil

${ }^{6}$ Department of Animal Science, Food and Nutrition, Southern Illinois University, Carbondale, IL, USA
}

\section{Corresponding Author:}

José R. Figueiredo, Laboratory of Manipulation of Oocytes and Preantral Follicles (LAMOFOPA), Faculty of Veterinary Medicine, State University of Ceara, Av Dr Silas Munguba, I700, Campus do Itaperi, Fortaleza, Ceara, Brazil. Email: jrf.lamofopapapers@gmail.com 
vitro maturation (IVM) and in vitro embryo culture (IVC) are some of the main approaches nowadays used for several research groups. ${ }^{4,5}$

Several factors may impair oocyte and embryo development in vitro, such as nutritional imbalances, hormonal disturbances, and oxidative stress. ${ }^{6,7}$ One of the main differences between the in vivo and in vitro environments is the high oxygen tension at which the oocytes are exposed in vitro, ${ }^{8,9}$ leading to increasing the production of reactive oxygen species (ROS).$^{10}$ An excessive amount of ROS induces mitochondrial dysfunction and causes damage in DNA, RNA, and proteins, ${ }^{11}$ as well as inhibits the spermoocyte fusion. ${ }^{12}$ In vivo, the mitochondria are a major source of ROS, such as superoxide anion radical, hydrogen peroxide, and hydroxyl radical; these radicals are produced endogenously by the proton electrochemical gradient during mitochondrial respiration. ${ }^{13}$ However, ROS production is balanced by endogenous antioxidant enzymes, such as superoxide dismutase (SOD), catalase (CAT), and glutathione peroxidase (GPX) ${ }^{14}$ Nevertheless, under in vitro conditions, oocytes and embryos have reduced antioxidant capacity, since in this system, they do not benefit from intrinsic antioxidant protection, ${ }^{6}$ making it extremely necessary to supplement culture media with antioxidants.

In order to protect oocytes and embryos from oxidative stress during IVM and IVC, different antioxidants have been tested, such as ascorbic acid, ${ }^{15}$ melatonin, ${ }^{16}$ cortisol,,${ }^{17}$ green tea, ${ }^{18}$ and anethole. ${ }^{19}$ The latter is the major component of the essential oil extracted from the plant Croton zehntneri (Euphorbiaceae), locally known as "canela de cunhã" or "canelinha," originating from northeastern Brazil. $^{20}$ The antioxidant activity of anethole has been confirmed in vitro in ML1-a cells ${ }^{21}$ and caprine preantral follicles ${ }^{19,22}$ and in vivo. ${ }^{23}$ Anethole has been proposed to act as a synergistic antioxidant increasing the activity of primary antioxidants (eg, SOD, CAT, and GPX), and the levels of tripeptide glutathione (GSH). ${ }^{24}$ Moreover, anethole has been shown to improve the survivability and development in vitro of isolated caprine secondary follicles. ${ }^{19}$ Despite the beneficial effect of anethole on in vitro culture of preantral follicles, to the best of our knowledge, there are no studies regarding its impact on oocyte IVM and IVC. Therefore, the present study aimed to investigate the effect of anethole supplementation to IVM media by evaluating the following end points: (1) oocyte maturation, (2) levels of ROS, (3) total antioxidant potential, (4) mitochondrial membrane potential, (5) fertilization rate, and (6) embryo development in cattle.

\section{Materials and Methods}

\section{Chemicals and Media}

Unless otherwise mentioned, the culture media, anethole, and other chemicals used in the present experiment were purchased from Sigma (St. Louis, Missouri).

\section{Collection of Oocytes}

Bovine ovaries were collected from a local abattoir immediately after slaughter, transported to the laboratory in $0.9 \%$ saline solution $(\mathrm{NaCl})$ supplemented with antibiotics $(100 \mu \mathrm{g} / \mathrm{mL}$ kanamycin sulphate) at $33^{\circ} \mathrm{C}$ to $35^{\circ} \mathrm{C}$ within 2 hours and washed twice in the laboratory with saline solution. ${ }^{25}$ The cumulus oocyte complexes (COCs) were collected from follicles (4-8 $\mathrm{mm})$ using an $18-\mathrm{G}$ needle connected to a $10-\mathrm{mL}$ disposable syringe. The COCs were selected in Dulbecco phosphate buffered saline (DPBS) supplemented with 5.56-mM glucose, 1.25 -mM sodium pyruvate, $15-\mu \mathrm{g} / \mathrm{mL}$ sodium heparin, $8-\mu \mathrm{g} / \mathrm{mL}$ phenol red, and $10-\mu \mathrm{g} / \mathrm{mL}$ gentamycin, under a stereomicroscope (X100 magnification; SMZ 645 Nikon, Tokyo, Japan; ). Only COCs with homogeneous and nondark cytoplasm, surrounded by 2 or more compact layers of cumulus cells, and an intact zona pellucida, were selected for IVM.

\section{In Vitro Maturation}

The selected COCs were washed twice in DPBS and 3 times in IVM medium without hormone, previously equilibrated for at least 3 hours at $38.5^{\circ} \mathrm{C}$ under $5 \% \mathrm{CO}_{2}$. The medium used for IVM was Tissue Culture Medium (TCM-199) supplemented with $10 \%$ fetal bovine serum (FBS), $0.2-\mathrm{mM}$ sodium pyruvate, 2-mM L-glutamine, $50-\mu \mathrm{g} / \mathrm{mL}$ gentamicin, $10-\mathrm{IU} / \mathrm{mL}$ equine chorionic gonadotrophin, and $10-\mathrm{IU} / \mathrm{mL}$ human chorionic gonadotrophin; this medium was referred to as TCM- $199^{+}$. For IVM, COCs were randomly allocated into 4 treatments as follows: TCM- $199^{+}$(control) or TCM-199 $9^{+}$supplemented with anethole at 30 (AN30), 300 (AN300), or $2000 \mu \mathrm{g} / \mathrm{mL}$ (AN2000). A total of 50 COCs per replicate were cultured in 4-well multidish (Nunc, Roskilde, Denmark) containing 500 $\mu \mathrm{L}$ of maturation medium, incubated for 24 hours at $38.5^{\circ} \mathrm{C}$ and $5 \% \mathrm{CO}_{2}$ in air. The experiment was replicated 6 times.

\section{Assessment of Oocyte Viability and Chromatin Configuration}

After IVM, COCs were mechanically denuded by repeated pipetting, and chromatin configuration was assessed by fluorescence microscopy (Nikon, Eclipse 80i, Tokyo, Japan). Oocytes were incubated for 30 minutes in $500 \mu \mathrm{L}$ of PBS supplemented with $0.5 \%$ of glutaraldehyde and $10-\mu \mathrm{M}$ hoechst 33342 (emission at $483 \mathrm{~nm}$ ). According to the chromatin configuration, oocytes were classified as: germinal vesicle, germinal vesicle breakdown, metaphase I, MII, or degenerated when chromatin showed abnormal configuration.

\section{In Vitro Fertilization}

Spermatozoa were obtained from frozen-thawed semen collected from one fertile bull. Thawed sperm were washed in a discontinuous gradient of $45 / 90 \%$ Percoll at $700 g$ for 30 minutes at room temperature, and the pellet was initially resuspended with $5-\mathrm{mL}$ Sperm-TALP. ${ }^{26}$ The final pellet was resuspended in in vitro fertilization (IVF)-TALP medium ${ }^{27}$ supplemented with $30-\mu \mathrm{g} / \mathrm{mL}$ 
heparin, $30-\mu \mathrm{g} / \mathrm{mL}$ penicillinamine, $15-\mu \mathrm{M}$ hypotaurine, and $1-\mu \mathrm{M}$ epinephrine. After 24 hours of IVM, one half of the COCs were processed for IVF and were washed twice in IVF-TALP medium, then transferred in groups of 50 to $500 \mu \mathrm{L}$ of IVFTALP medium containing $2 \times 10^{6}$ spermatozoa/mL in 4 well Nunc multidishes. Spermatozoa and COCs were incubated for 18 hours at $38.5^{\circ} \mathrm{C}$ in a humidified atmosphere of $5 \% \mathrm{CO}_{2}$ in air.

\section{In Vitro Culture}

Presumptive zygotes were removed from the IVF-TALP medium, mechanically denuded by repeated pipetting, and washed twice in synthetic oviductal fluid (SOF) medium supplemented with Basal Medium Eagle (BME) amino acids solution 50X, MEM amino acids solution 100X, and 5\% FBS. Groups of 20 presumptive zygotes were transferred to $20-\mu \mathrm{L}$ drops of SOF medium under mineral oil in a $30-\mathrm{mm}$ Petri dish and cultured for 7 days at $38.5^{\circ} \mathrm{C}$ in a humidified atmosphere of $5 \% \mathrm{CO}_{2}$ in air. Half of the medium was replaced every 2 days. Cleavage was evaluated on day 3 after IVF (day $0=$ IVF), and blastocysts were evaluated on day 7 .

\section{Reactive Oxygen Species Levels}

The levels of ROS in the culture medium were measured by a spectrofluorimetric method, ${ }^{28}$ using $2^{\prime}, 7^{\prime}$-dihydrodichlorofluorescein diacetate (DCHF-DA) assay. Sample aliquot $(50 \mu \mathrm{L})$ was incubated with $10 \mu \mathrm{L}$ of DCHF-DA $(1 \mathrm{mM})$. The oxidation of DCHF-DA to fluorescent dichlorofluorescein (DCF) was measured for the detection of ROS. The DCF fluorescence intensity (FI) emission was recorded at $520 \mathrm{~nm}$ (with $480 \mathrm{~nm}$ excitation) 2 hours after the addition of DCHF-DA to the culture medium.

\section{Evaluation of Total Antioxidant Potential}

The total antioxidant potential was determined by the ferric reducing antioxidant power (FRAP) assay. ${ }^{29}$ In the FRAP assay, the antioxidant potential in the culture medium was evaluated by measuring the conversion/reduction of $\mathrm{Fe}^{3+}$ to $\mathrm{Fe}^{2+}$, which is chelated by 2,4,6-Tri (2-pyridyl) S-triazine (TPTZ) to form the $\mathrm{Fe}^{2+}$-TPTZ complex, with a maximum absorbance at $593 \mathrm{~nm}$. A curve with ascorbic acid was used as a positive control.

\section{Oocyte Mitochondrial Membrane Potential and Mitochondrial ROS Production Assay}

Oocytes from every treatment were assessed by fluorescent probes for mitochondrial membrane potential (MitoTracker ${ }^{\circledR}$ Orange, Molecular Probes ${ }^{\circledR}$; Invitrogen, Ltd, Paisley United Kingdom), ${ }^{30}$ and ROS (CM-H2DCFDA). ${ }^{31}$ For mitochondrial membrane potential, oocytes were incubated in $2 \mu \mathrm{L} / \mathrm{mL}$ MitoTracker ${ }^{\circledR}$ Orange for 30 minutes at $37^{\circ} \mathrm{C}$. For ROS assay, oocytes were incubated in $1 \mu \mathrm{L} / \mathrm{mL}$ H2DCFDA for 30 minutes. Six oocytes/ treatment were used for each of the 2 assays described above.

After the incubation period of each fluorescent probe, oocytes were fixed in glutaraldehyde at room temperature for 15 minutes, and then transferred to PBS solution for up to 1 hour at room temperature protected from light for fluorescent image analysis. Oocytes were mounted on a slide for confocal laser scanning microscopy analysis (Zeiss LSM 710, Oberkochen, Germany). For each sample, images with $1024 \times 1024$ pixels were drawn to measure the FI. Parameters related to FI, such as laser energy, signal detection (gain), and pinhole size, were maintained at constant values for all measurements. Oocytes were observed at $40 \times$ objective magnification under oil immersion. A helium/neon laser ray at $543 \mathrm{~nm}$ (551-nm excitation and 576-nm emission) was used to identify the MitoTracker ${ }^{\circledR}$ Orange. An argon ion laser ray at $488 \mathrm{~nm}$ (495-nm excitation and 519-nm emission) was used to identify the H2DCFDA. Scanning was conducted with $\mathrm{Z}$ stack of 25 optical series from the top to the bottom of the oocyte with a step size of $0.08 \mathrm{~mm}$ to allow 3-dimensional distribution analysis.

\section{Statistical Analyses}

Statistical analyses were performed using Sigma Plot 11 (Systat Software Inc, San Jose, California). Comparison of means among treatments was analyzed by 1-way analysis of variance and Tukey test. The MII and cleavage and embryo development rates were analyzed by $\chi^{2}$ test. Pearson correlation test was used to evaluate the association between MitoTracker levels and cleavage rates. Data are presented as a mean $( \pm$ SEM) and percentage, and the statistical significance was set at $P<.05$.

\section{Results}

\section{Effects of Anethole Supplementation on the Maturation Potential of Oocytes}

To investigate the effects of anethole on oocytes in MII stage and after fertilization, oocytes were examined after IVM in the presence or absence of different anethole concentrations (Figure 1A and B). After IVM, all oocytes resumed meiosis. The percentages of MII oocytes were similar $(P>.05)$ among the treatments and ranged from $77.3 \%$ to $95.7 \%$. However, after IVF, the addition of anethole at $2000 \mu \mathrm{g} / \mathrm{mL}$ to the control medium increased $(P<.05)$ the percentage of penetrated oocytes when compared to the control treatment only.

\section{Levels of ROS and FRAP After IVM}

The levels of ROS and FRAP measured in the culture medium after IVM are shown (Figure 2). Although ROS levels were similar among all treatments, the levels of FRAP were higher $(P<$ $.05)$ in the AN300 treatment compared to the control treatment.

\section{Mitochondrial Membrane Potential and ROS Production Levels in the Oocyte}

The levels of ROS (H2DCF-DA) and the mitochondrial membrane potential (MitoTracker ${ }^{\circledR}$ Orange) in the oocyte after IVM 

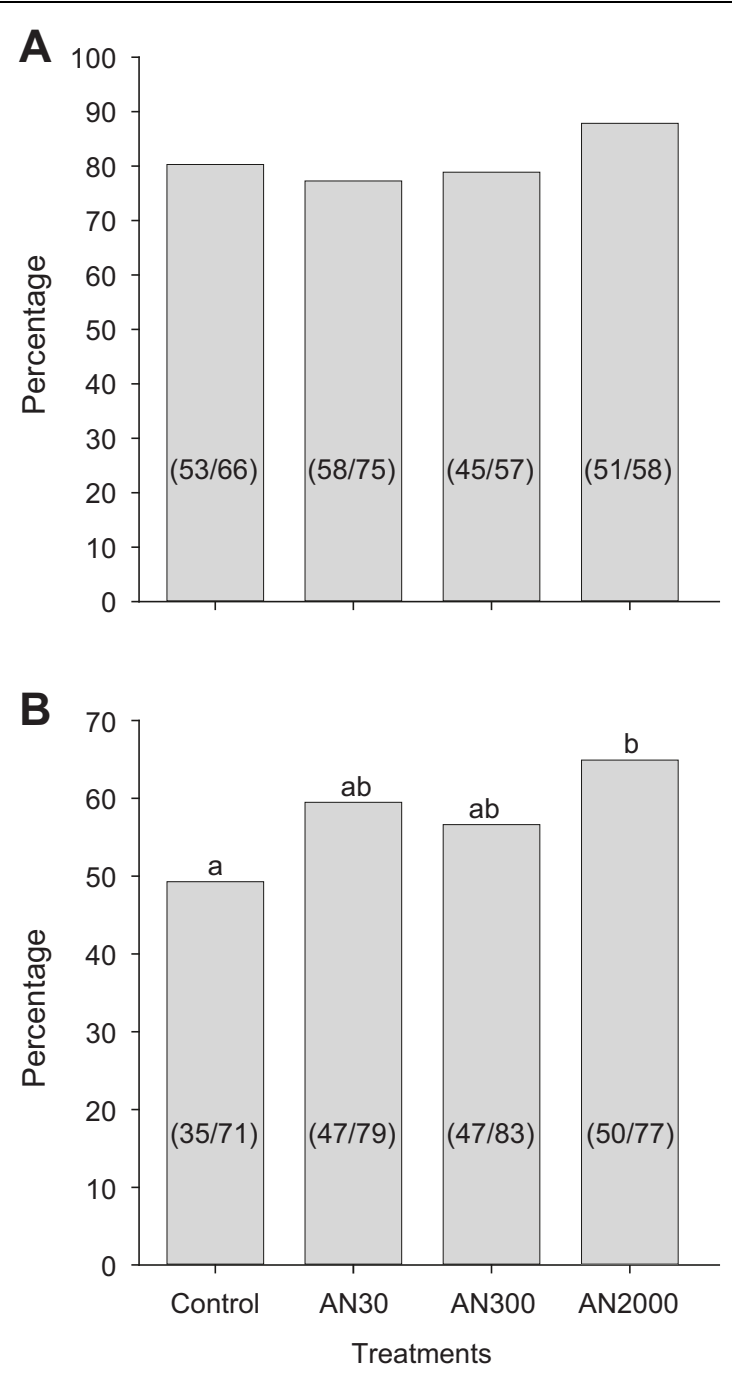

Figure I. Nuclear maturation (A) and fertilization (B) rates in bovine oocytes after in vitro maturation in control medium with or without supplementation of anethole at 30 (AN30), 300 (AN300), or $2000 \mu \mathrm{g} / \mathrm{mL}$ (AN2000). ${ }^{\mathrm{a}, \mathrm{b}}$ Different letters indicate significant differences among treatments $(P<.05)$.

are shown (Figures 3 and 4, respectively). The ROS production in the oocytes after maturation did not differ $(P>.05)$ among treatments. However, oocytes treated with anethole at $300 \mu \mathrm{g} /$ $\mathrm{mL}$ had higher $(P<.05)$ mitochondrial membrane potential when compared to the control treatment \pm 0.2 vs $15.3 \pm$ 2.9). In addition, a positive association between mitochondrial membrane potential and cleavage rate was observed by correlation analysis (Figure 5).

\section{Effects of Anethole Addition to the IVM Medium on In Vitro Embryo Development}

The impact of anethole addition to the IVM medium on embryo development is shown (Table 1). Anethole at $300 \mu \mathrm{g} / \mathrm{mL}$ was the only treatment that yielded higher $(P<.05)$ cleavage rate and embryo development (morula + blastocyst) production

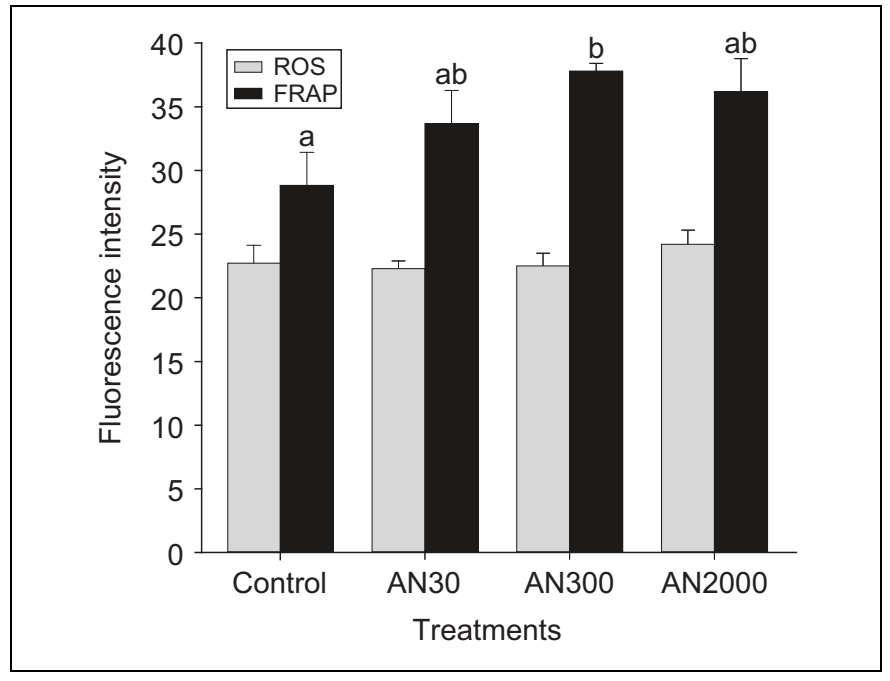

Figure 2. Fluorescence intensity of ROS and FRAP in the culture medium after in vitro maturation of bovine oocytes in control medium with or without supplementation of anethole at 30 (AN30), 300 (AN300), or $2000 \mu \mathrm{g} / \mathrm{mL}$ (AN2000). ${ }^{\mathrm{a}, \mathrm{b}}$ Different letters indicate significant differences among treatments $(P<.05)$. FRAP indicates ferric reducing antioxidant power; ROS, reactive oxygen species.

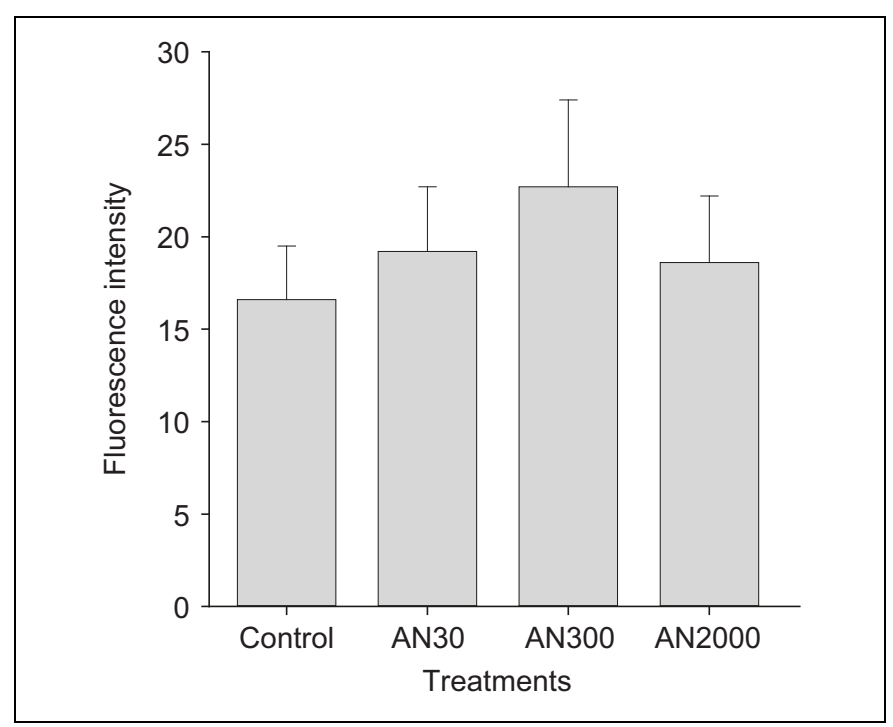

Figure 3. Mean ( \pm SEM) fluorescence units of mitochondrial ROS after in vitro maturation of bovine oocytes in control medium with or without supplementation of anethole at 30 (AN30), 300 (AN300), or $2000 \mu \mathrm{g} / \mathrm{mL}$ (AN2000). No difference among treatments was observed. ROS indicates reactive oxygen species.

compared to the control treatment. Furthermore, the AN300 treatment had greater $(P<.05)$ average number of total cells in blastocysts compared to the control and AN30 treatments.

\section{Discussion}

This study shows for the first time that anethole added to IVM medium, despite not affecting oocyte nuclear maturation, increased in a concentration-dependent manner the total 


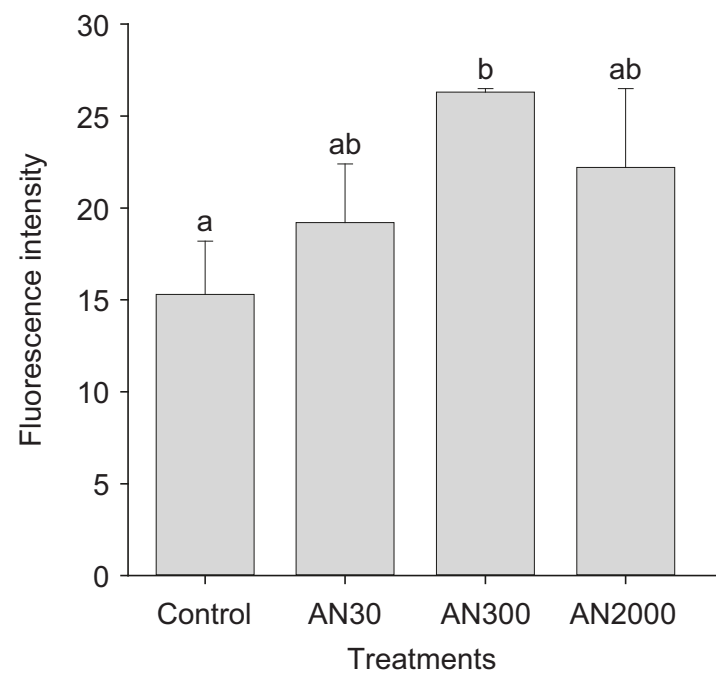

Figure 4. Mean ( \pm SEM) fluorescence units of mitochondrial membrane potential after in vitro maturation of bovine oocytes in control medium with or without supplementation of anethole at 30 (AN30), 300 (AN300), or $2000 \mu \mathrm{g} / \mathrm{mL}$ (AN2000). ${ }^{\mathrm{a}, \mathrm{b}}$ Different letters indicate significant differences among treatments $(P<.05)$.

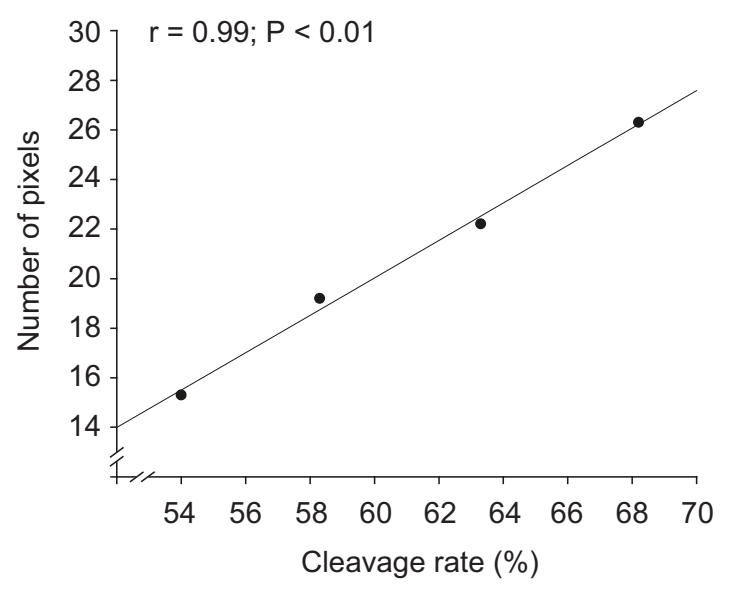

Figure 5. Correlation between oocyte mitochondrial membrane potential (number of pixels) and percentage of embryo cleavage rate. Each dot represents a treatment with their respective oocyte mitochondrial membrane potential versus its overall cleavage rate.

antioxidant potential and mitochondria membrane potential of bovine oocytes and improved the quantity and quality(based on the number of embryonic cells) of in vitro produced embryos. Previous studies by our team demonstrated the protective effect of anethole against oxidative damage when added to culture medium of goat preantral follicles cultured either in situ or in the isolated form. ${ }^{19,22}$

In the present study, supplementing the IVM medium with anethole did not result in a higher proportion of oocytes that reached the MII stage. Likewise, other antioxidants have shown similar results when added to bovine IVM medium, that is, no effect on oocyte chromatin configuration. ${ }^{32,33}$ In this
Table I. In Vitro Embryo Development From Matured Bovine Oocytes in Control Medium With or Without Supplementation of Anethole at 30 (AN30), 300 (AN300), or AN2000. ${ }^{\mathrm{a}}$

\begin{tabular}{lcccc}
\hline Treatment & $\mathrm{n}$ & $\begin{array}{c}\text { Cleavage } \\
\text { Rate (\%) }\end{array}$ & $\begin{array}{c}\text { Embryo } \\
\text { Development (\%) }\end{array}$ & $\begin{array}{c}\text { No. Embryo } \\
\text { Cells }\end{array}$ \\
\hline Control & 100 & $54.0(54)^{\mathrm{c}}$ & $13.0(13)^{\mathrm{c}}$ & $119.9 \pm 11.3^{\mathrm{c}}$ \\
AN30 & 108 & $58.3(63)^{\mathrm{c}, \mathrm{d}}$ & $17.6(19)^{\mathrm{c}, \mathrm{d}}$ & $124.5 \pm 5.1^{\mathrm{c}}$ \\
AN300 & 107 & $68.2(73)^{\mathrm{d}}$ & $27.1(29)^{\mathrm{d}}$ & $144.1 \pm 3.1^{\mathrm{d}}$ \\
AN2000 & 109 & $63.3(69)^{\mathrm{c}, \mathrm{d}}$ & $18.3(20)^{\mathrm{c}, \mathrm{d}}$ & $130.8 \pm 4.1^{\mathrm{c}, \mathrm{d}}$ \\
\hline
\end{tabular}

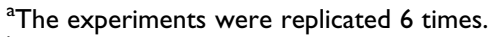

bMorula + blastocyst.

$c, d$ Within a column, different letters indicate significant differences among treatments $(P<.05)$.

regard, it has been suggested that intracellular antioxidant content at the end of IVM did not appear to affect oocyte nuclear maturation, but rather exerted a positive effect on cytoplasmic maturation and, consequently, on early embryonic development. ${ }^{3}$

After IVF, oocytes matured in vitro in the AN2000 treatment had a significant increase in the fertilization rate (ie, percentage of oocytes with 2 pronuclei formation, male and female). Gamete fusion induces the release of free calcium $\left(\mathrm{Ca}^{2+}\right)$ within the mammalian oocyte. Multiple $\mathrm{Ca}^{2+}$ oscillations trigger an orderly sequence of events that include the release of cortical granules, which prevent polyspermy, the completion of the second meiotic division, and the formation of the female pronucleus. ${ }^{34}$ Anethole has been suggested as a trigger that may induce the opening of voltage-dependent $\mathrm{Ca}^{2+}$ channels. ${ }^{35}$ Therefore, we believe that anethole at the concentration of $2000 \mu \mathrm{g} / \mathrm{mL}$ may have improved normal fertilization rates by opening $\mathrm{Ca}^{2+}$ channels, thus triggering the events necessary for pronuclei formation.

The addition of anethole during IVM altered neither the intra- nor extracellular ROS concentrations; nevertheless, the AN300 treatment increased the total antioxidant potential. The balance between ROS formation and elimination is known as the redox balance. In the oocyte, the main ROS scavenger system is GSH in the reduced state, and glutathione disulfide in the oxidized state. Glutathione uses a reducing power provided by oxidative metabolism, tied to redox couples such as $\mathrm{NADPH} / \mathrm{NADP}^{+}$, to counteract oxidation via ROS. ${ }^{36}$ Any unbalance in this redox state may have deleterious effects on cells. ${ }^{37}$ It has been previously reported that anethole may play an important role in the maintenance of the redox balance by either reducing ROS levels ${ }^{22}$ or inducing the synthesis of cellular antioxidants such as CAT, SOD,${ }^{38}$ and GPX. ${ }^{21}$ Accordingly, our results suggest that anethole improved the redox balance by increasing the total oocyte antioxidant capacity after maturation.

Interestingly, oocytes from the AN300 treatment had a higher mitochondria membrane potential. Mitochondria play a central role in energy production, cell metabolism, and cell death and are the main generator of free radicals in mammals. ${ }^{39}$ For this reason, the mitochondrial pattern has been associated 
with the quality and developmental capacity of mammalian oocytes and embryos. ${ }^{40}$ Previous reports have shown that mitochondria membrane potential is lower in immature oocytes compared to the mature ones and that mitochondrial activity is related to oocyte competence. ${ }^{41,42}$ Based on our findings of the mitochondria membrane potential and redox balance, we can suggest that anethole positively influenced oocyte cytoplasmic maturation in a concentration-dependent manner since higher rates of embryo production were obtained.

Regarding IVP, oocytes exposed to $300 \mu \mathrm{g} / \mathrm{mL}$ anethole during IVM showed higher rates of cleavage, embryonic development, and total cell number per blastocyst. It is well known that ROS levels increase, ${ }^{43}$ and low adenosine triphosphate (ATP) production $^{44}$ can severely affect embryonic development in vitro. Variations in ROS concentration can affect the expression of several genes, including those for protein kinases, tyrosine kinases, and growth factors. ${ }^{45}$ However, it has been suggested that redox balance rather than ROS ensures appropriate levels of gene expression related to cell cycle regulation. ${ }^{46}$ In this regard, early embryo development and implantation potential have been correlated with mitochondrial function and activity. ${ }^{4,48}$ The malfunction of the mitochondria generates a decrease in cellular ATP content that generally leads to cell apoptosis. ${ }^{49}$ Here, we found a positive correlation between mitochondria membrane potential and cleavage rates. Therefore, the improvements in IVP in this study can be attributed to both the higher redox capacity and the higher mitochondrial membrane potential of the in vitro matured oocytes in the presence of anethole.

In conclusion, our results demonstrate that supplementing the IVM medium with $300 \mu \mathrm{g} / \mathrm{mL}$ anethole improves the quantity and quality of bovine embryos produced in vitro. Moreover, the beneficial effects of anethole on embryonic developmental competence may have been caused by its capacity to regulate the redox balance and improve mitochondrial function.

\section{Acknowledgments}

The authors are grateful to Central Analítica UFC/CT-INFRA/MCTISISNANO/Pró- Equipamentos, UFC Department of Physics for the technical assistance.

\section{Declaration of Conflicting Interests}

The author(s) declared the following potential conflicts of interest with respect to the research, authorship, and/or publication of this article. Naiza Arcângela R. Sá is the recipient of a doctoral scholarship from CAPES.

\section{Funding}

The author(s) disclosed receipt of the following financial support for the research, authorship, and/or publication of this article: This work was supported by the National Council for Scientific and Technological Development (CNPq; Grant \# 407594/2013-2) and Coordination for the Improvement of Higher Education Personnel (CAPES).

\section{References}

1. Arias-Alvarez M, Bermejo-Alvarez P, Gutierrez-Adan A, Rizos D, Lorenzo PL, Lonergan P. Effect of leptin supplementation during in vitro oocyte maturation and embryo culture on bovine embryo development and gene expression patterns. Theriogenology. 2011;75(5):887-896.

2. Lonergan P, Fair T. Maturation of oocytes in vitro. Annu Rev Anim Biosci. 2016;4:255-268.

3. Chowdhury MMR, Mesalam A, Khan I, et al. Improved developmental competence in embryos treated with lycopene during in vitro culture system. Mol Reprod Dev. 2018;85(1):46-61.

4. Zhu J, Moawad AR, Wang CY, Li HF, Ren JY, Dai YF. Advances in in vitro production of sheep embryos. Int J Vet Sci Med. 2018;6: 15-26.

5. Figueira RCS, Setti AS, Braga DPAF, Iaconelli A Jr, Borges E Jr, . Blastocyst morphology holds clues concerning the chromosomal status of the embryo. Int J Fertil Steril. 2015;9(2):215-220.

6. Combelles CM, Gupta S, Agarwal A. Could oxidative stress influence the in vitro maturation of oocytes? Reprod Biomed Online. 2009; 18(6):864-880.

7. Murray AA, Swales AK, Smith RE, Molinek MD, Hillier SG, Spears N. Follicular growth and oocyte competence in the in vitro cultured mouse follicle: effects of gonadotrophins and steroids. Mol Hum Reprod. 2008;14(2):75-83.

8. Tilly JL, Tilly KI. Inhibitors of oxidative stress mimic the ability of follicle stimulating hormone to suppress apoptosis in cultured rat ovarian follicles. Endocrinology. 1995;136(1):242-252.

9. Hovatta O, Silye R, Abir R, Krausz T, Winston RM. Extracellular matrix improves survival of both stored and fresh human primordial and primary ovarian follicles in long-term culture. Hum Reprod. 1997;12(5):1032-1036.

10. Agarwal A, Said TM, Bedaiwy MA, Banerjee J, Alvarez JG. Oxidative stress in an assisted reproductive technique setting. Fertil Steril. 2006;86(3):503-512.

11. Finkel T, Holbrook NJ. Oxidants, oxidative stress and the biology of ageing. Nature. 2000;408(6809):239-247.

12. Aitken RJ, Harkiss D, Buckingham D. Relationship between iron catalysed lipid peroxidation potential and human sperm function. J Reprod Fertil. 1993;98(1):257-265.

13. Takahashi T, Igarashi H, Amita M, Hara S, Matsuo K, Kurachi H. Molecular mechanism of poor embryo development in postovulatory aged oocytes: mini review. J Obstet Gynaecol Res. 2013; 39(10):1431-1439.

14. He L, He T, Farrar S, Ji L, Liu T, Ma X. Antioxidants maintain cellular redox homeostasis by elimination of reactive oxygen species. Cell Physiol Biochem. 2017;44(2):532-553.

15. Andrade ER, van den Hurk R, Lisboa LA, et al. Effects of ascorbic acid on in vitro culture of bovine preantral follicles. Zygote. 2012;20(4):379-388.

16. Cebrian-Serrano A, Salvador I, Raga E, Dinnyes A, Silvestre MA. Beneficial effect of melatonin on blastocyst in vitro production from heat-stressed bovine oocytes. Reprod Domest Anim. 2013; 48(5):738-746.

17. da Costa NN, Brito KN, Santana PD, et al. Effect of cortisol on bovine oocyte maturation and embryo development in vitro. Theriogenology. 2016;85(2):323-329.

18. Wang Z, Fu C, Yu S. Green tea polyphenols added to IVM and IVC media affect transcript abundance, apoptosis, and pregnancy rates in bovine embryos. Theriogenology. 2013;79(1):186-192. 
19. Sá NAR, Araújo VR, Correia HHV, et al. Anethole improves the in vitro development of isolated caprine secondary follicles. Theriogenology. 2017;89:226-234.

20. Leal-Cardoso JH, Fonteles MC. Pharmacological effects of essential oils plants of the Northeast of Brazil. An Acad Bras Cienc. 1999;71(2):207-213.

21. Chainy GB, Manna SK, Chaturvedi MM, Aggarwal BB. Anethole blocks both early and late cellular responses transduced by tumor necrosis factor: effect on NF-kappaB, AP-1, JNK, MAPKK and apoptosis. Oncogene. 2000;19(25):2943-2950.

22. Sá NAR, Bruno JB, Guerreiro DD, et al. Anethole reduces oxidative stress and improves in vitro survival and activation of primordial follicles. Braz J Med Biol Res. 2018;51(8):7129-7137.

23. Freire RS, Morais SM, Catunda-Junior FE, Pinheiro DC. Synthesis and antioxidant, anti-inflammatory and gastroprotector activities of anethole and related compounds. Bioorg Med Chem. 2005; 13(13):4353-4358.

24. Khan A, Ahmad A, Akhtar F, et al. Induction of oxidative stress as a possible mechanism of the antifungal action of three phenylpropanoids. FEMS Yeast Res. 2011;11(1):114-122.

25. Takahashi Y, Hishinuma M, Matsui M, Tanaka H, Kanagawa H. Development of in vitro matured/fertilized bovine embryos in a chemically defined medium: influence of oxygen concentration in the gas atmosphere. J Vet Med Sci. 1996;58(9):897-902.

26. Papa PM, Papa FO, Oliveira LA, Guasti PN, Castilho C, Giometti IC. Different extenders in the cryopreservation of bovine epididymal spermatozoa. Anim Reprod Sci. 2015;161:58-63.

27. Gordon I. Laboratory Production of Cattle Embryos. Wallingford, England: CAB International; 1994.

28. Loetchutinat S, Kothan S, Dechsupa J, Meesungnoen J, Jay-Gerin SM. Spectrofluorometric determination of intracellular levels of reactive oxygen species in drug-sensitive and drug-resistant cancer cells using the 20,70-dichlorofluorescein diacetate assay. Radiat Phys Chem. 2005;72(2-3):323-331.

29. Benzie IFF, Strains JJ. The ferric reducing ability of plasma (FRAP) as a measure of "antioxidant power": the FRAP assay. Anal Biochem. 1996;239(1):70-76.

30. Perry SW, Norman JP, Barbieri J, Brown EB, Gelbard HA. Mitochondrial membrane potential probes and the proton gradient: a practical usage guide. Biotechniques. 2011;50(2): 98-115.

31. Fabbri R, Vicenti R, Martino NA, et al. Confocal laser scanning microscopy analysis of bioenergetic potential and oxidative stress in fresh and frozen-thawed human ovarian tissue from oncologic patients. Fertil Steril. 2014;101(3):795-804.

32. Chankitisakul V, Somfai T, Inaba Y, Techakumphu M, Nagai T. Supplementation of maturation medium with L-carnitine improves cryo-tolerance of bovine in vitro matured oocytes. Theriogenology. 2013;79(4):590-598.

33. Furnus CC, de Matos DG, Picco S, et al. Metabolic requirements associated with GSH synthesis during in vitro maturation of cattle oocytes. Anim Reprod Sci. 2008;109(1-4):88-99.
34. Clift D, Schuh M. Restarting life: fertilization and the transition from meiosis to mitosis. Nat Rev Mol Cell Biol. 2013;14(9): 549-562.

35. Soares PM, Lima RF, de Freitas Pires A, Souza EP, Assreuy AM, Criddle DN. Effects of anethole and structural analogues on the contractility of rat isolated aorta: involvement of voltagedependent $\mathrm{Ca}^{2+}$-channels. Life Sci. 2007;81(13):1085-1093.

36. Dumollard R, Ward Z, Carroll J, Duchen MR. Regulation of redox metabolism in the mouse oocyte and embryo. Development. 2007;134(3):455-465.

37. Pamplona R, Costantini D. Molecular and structural antioxidant defenses against oxidative stress in animals. Am J Physiol Regul Integr Comp Physiol. 2011;301(4):843-863.

38. Dongare V, Kulkarni C, Kondawar M, Magdum C, Haldavnekar $\mathrm{V}$, Arvindekar A. Inhibition of aldose reductase and anti-cataract action of trans-anethole isolated from Foeniculum vulgare Mill. fruits. Food Chem. 2012;132(1):385-390.

39. Brand MD, Orr AL, Perevoshchikova IV, Quinlan CL. The role of mitochondrial function and cellular bioenergetics in ageing and disease. Br J Dermatol. 2013;169(2):1-8.

40. Egerszegi I, Alm H, Rátky J, Heleil B, Brüssow KP, Torner H. Meiotic progression, mitochondrial features and fertilisation characteristics of porcine oocytes with different G6PDH activities. Reprod Fertil Dev. 2010;22(5):830-838.

41. Van Blerkom J, Davis P, Mathwig V, Alexander S. Domains of high-polarized and low-polarized mitochondria may occur in mouse and human oocytes and early embryos. Hum Reprod. 2002;17(2):393-406.

42. Tarazona AM, Rodríguez JI, Restrepo LF, Olivera-Angel M. Mitochondrial activity, distribution and segregation in bovine oocytes and in embryos produced in vitro. Reprod Domest Anim. 2006;41(1):5-11.

43. Goto Y, Noda Y, Mori T, Nakano M. Increased generation of reactive oxygen species in embryos cultured in vitro. Free Radic Biol Med. 1993;15(1):69-75.

44. Yu Y, Dumollard R, Rossbach A, Lai FA, Swann K. Redistribution of mitochondria leads to bursts of ATP production during spontaneous mouse oocyte maturation. J Cell Physiol. 2010; 224(3):672-680.

45. Nose K. Role of reactive oxygen species in the regulation of physiological functions. Biol Pharm Bull. 2000;23(8):897-903.

46. Day RM, Suzuki YJ. Cell proliferation, reactive oxygen and cellular glutathione. Dose Response. 2005;3(3):425-442.

47. Benkhalifa M, Ferreira YJ, Chahine H, et al. Mitochondria: participation to infertility as source of energy and cause of senescence. Int J Biochem Cell Biol. 2014;55:60-64.

48. Pang W, Zhang Y, Zhao N, Darwiche SS, Fu X, Xiang W. Low expression of Mfn2 is associated with mitochondrial damage and apoptosis in the placental villi of early unexplained miscarriage. Placenta. 2013;34(7):613-618.

49. Vander Heiden MG, Cantley LC, Thompson CB. Understanding the Warburg effect: the metabolic requirements of cell proliferation. Science. 2009;324(5930):1029-1033. 